\title{
A Remark on the Decomposition of Abelian Fiber Spaces over Curves
}

\author{
By \\ Yoshio Fujimoto*,**
}

\section{$\S 0$. Introduction}

By an Abelian fiber space $f: X \rightarrow Y$, we mean that $f$ is a proper surjective morphism from a complex manifold $X$ to a complex manifold $Y$ whose general fibers are Abelian varieties. If $g: X \rightarrow Y$ is a surjective meromorphic map of complex varieties with irreducible general fibers, $g$ is called a meromorphic fiber space. Using his Hodge theory with degenerating coefficients, Zucker [3] proved the theorem of the fixed part of Abelian varieties. ([3] Corollary 10.2) Inspired by his result, we consider the decomposition of Abelian fiber spaces over curves.

The purpose of this note is to prove the following Theorem A.

\section{Theorem (A).}

Let $f: X \rightarrow C$ be a surjective morphism of a projective manifold $X$ to a nonsingular curve $C$ such that the general fibers of $f$ are Abelian varieties. Then we have the following diagram

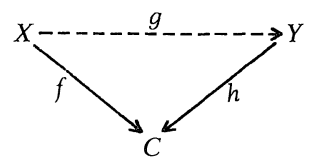

which enjoys the following properties.

(1) $g: X \rightarrow Y$ (resp. $h: Y \rightarrow C$ ) is a meromorphic fiber space (resp. a fiber space) whose general fibers are Abelian varieties and the period map associated to $h$ is constant.

(For the definition of a fiber space, a meromorphic fiber space and a period map, see notations below.)

(2) Suppose that there exists a commutative diagram

Communicated by K. Saito, July 1, 1988. Revised December 1, 1988.

* Department of Mathematics, Kyoto University, Kyoto 606, Japan.

Current address: Department of Mathematics, Shizuoka University, Ohya, Shizuoka, Japan.

** Supported by the JSPS Fellowships for Japanese Junior Scientists 


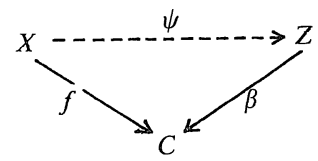

where $\psi: X \rightarrow Z$ (resp. $\beta: Z \rightarrow C$ ) is a meromorphic fiber space (resp. a fiber space) whose general fibers are Abelian varieties and the period map associated to $\beta$ is constant. Then there exists a unique meromorphic map $\varphi: Y \rightarrow Z$ such that we obtain the following commutative diagram. Moreover we have $\operatorname{dim} H^{1}\left(X, \mathcal{O}_{X}\right)$ $=\operatorname{dim} H^{1}\left(Y, \mathcal{O}_{Y}\right)$.

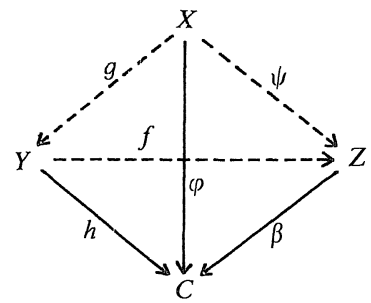

The author wishes to express his thanks to Professor $\mathbf{K}$. Ueno for useful suggestions.

Notations. We use the following convention and terminology.

$$
q(X):=\operatorname{dim} H^{1}\left(X, \mathcal{O}_{X}\right): \text { the irregularity of } X .
$$

A fiber space is a proper surjective morphism of complex spaces with irreducible general fibers.

Let $f: X \rightarrow Y$ be a meromorphic map of complex varieties, $\Gamma \subset X \times Y$ be the graph of $f, p^{\prime}: \Gamma \rightarrow Y$ be the natural projection. If $p^{\prime}$ is a fiber space, we say that $g$ is a meromorphic fiber space.

Let $f: X \rightarrow C$ be an Abelian fiber space over the curve $C$ and let $f^{-1}(p)$ $=\sum_{i=1}^{\lambda} m_{i} D_{i}$ be the irreducible decomposition of the singular fiber over a point $p \in C$. If the greatest common divisor $m$ of $\left(m_{1}, \cdots, m_{\lambda}\right)$ is greater than one, we say that $X$ has multiple fibers of multiplicity $m$ at $p \in C$.

Let $\varphi: X \rightarrow Y$ be a projective family of $g$-dimensional Abelian varieties and $U \subset Y$ be a Zariski open subset over which $\varphi$ is smooth. Then by integrating a relative holomorphic one-form of $X$ along a continuous family of homology basis of $H_{1}\left(\varphi^{-1}(u), \mathbb{Z}\right), u \in U$, we can define a multi-valued holomorphic mapping (called the multi-valued period mapping) $T: U \rightarrow \subseteq_{g}$ into the Siegel upper halfplane of degree $g$. For the precise definition of a period mapping, see Ueno [2], p.4-8.

\section{\$1. Preliminaries}

To prove Theorem A, we need the following propositions. 
Proposition 1.1. Let $f: X \rightarrow C$ be an Abelian fiber space over a smooth curve $C$ and define $q(f):=q(X)-q(C)$. Then we have the inequality $q(f) \leq \operatorname{dim}(X)-1$.

Proposition 1.2. Let $f: X \rightarrow C$ be a proper surjective morphism of a projective manifold $X$ to a smooth curve $C$ such that the general fibers are Abelian varieties. Then we have the following diagram

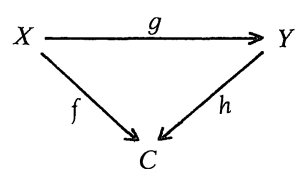

which satisfies the following properties.

(1) $g: X \rightarrow Y$ and $h: Y \rightarrow C$ are Abelian fiber spaces. Moreover we have $q(X)=q(Y)$ and $h: Y \rightarrow C$ is a Seifert fiber space, that is, a fiber space with constant moduli which has only multiple singular fibers.

(2) Suppose that there exists a commutative diagram

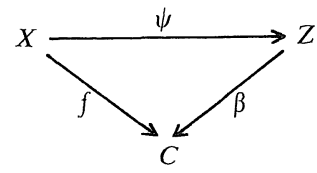

such that $\psi: X \rightarrow Z$ and $\beta: Z \rightarrow C$ are Abelian fiber spaces with $q(X)$ $=q(Z)$. Then there exists a unique morphism $\varphi: Z \rightarrow Y$ with the following commutative diagram.

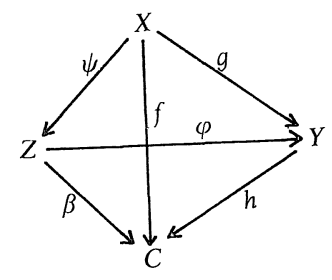

Proof of Proposition 1.1 and Proposition 1.2.

Let $\alpha_{X}$ be the Albanese map of $X$ and consider the following commutative diagram:

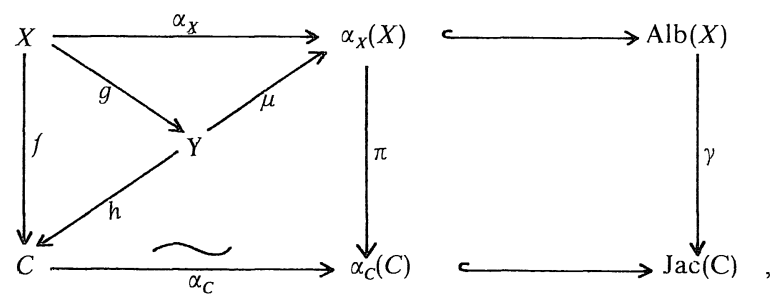


where $\mu \circ g$ is the Stein factorization of $\alpha_{X}$.

The Albanese map $\alpha_{X}: X \rightarrow A l b(X)$, when restricted to the general fiber of $f$, is a Lie group homomorphism, hence each fiber of $\pi$ and the general fiber of $g$ are Abelian varieties. And it is easy to see that the general fibers of $h$ are Abelian varieties.

As $\alpha_{X}(X)$ generates $A l b(X)$, for a general point $p \in C, \alpha_{X}\left(f^{-1}(p)\right)$ is equal to the connected component of $\operatorname{ker}\{\gamma: \operatorname{Alb}(X) \rightarrow \operatorname{Jac}(C)\}$. Hence we have $\operatorname{dim}(Y)$ $=\operatorname{dim}\left(\alpha_{X}(X)\right)=1+q(X)-q(C)$. This proves Proposition 1.1. Moreover we have $q(X)=q(Y)$, since $q(X) \geq q(Y) \geq q(\alpha(X))=q(X)$. Each fiber of $h$ contains no rational curve and the ramification locus of $\mu$ are at most multiple fibers of $h$. Hence $h: Y \rightarrow C$ is a Seifert fiber space.

Universality. Suppose that there exists a commutative diagram

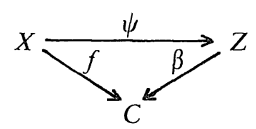

with $q(X)=q(Z)$. Then we have the following commutative diagram:

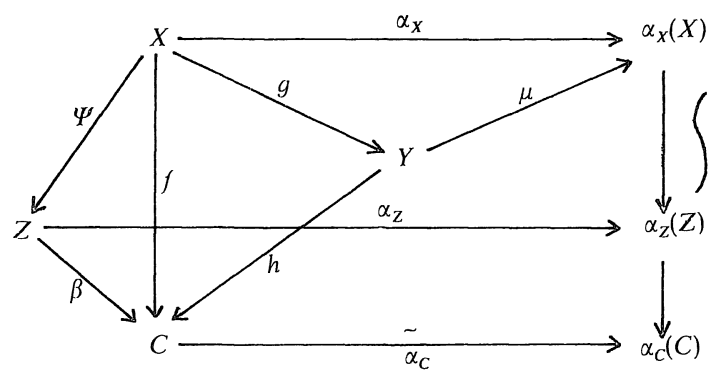

Since $q(X)=q(Y)=q(Z)$, we have $\alpha_{X}(X) \simeq \alpha_{Y}(Y) \simeq \alpha_{Z}(Z)$. Hence for each fiber $X_{p}:=\psi^{-1}(p)$ of $\psi, \mu \circ g\left(X_{p}\right)=\alpha_{X}\left(X_{p}\right)$ consists of one point. Since $\mu$ is finite and $X_{p}$ is connected, $g\left(X_{p}\right)$ is also a point. Hence there exists a holomorphic map $\varphi: Z \rightarrow Y$ such that $g$ is isomorphic to $\varphi^{\circ} \psi$.

q.e.d.

\section{Lemma 1.3. Let}

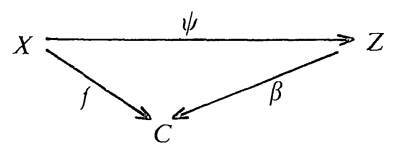

be a commutative diagram of Abelian fiber spaces over a smooth curve, where the Albanese map of $Z$ is generically finite to its image. And let 


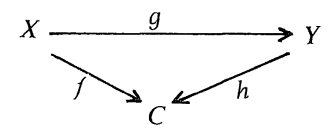

be a commutative diagram of Abelian fiber spaces as in Proposition 1.2.

Then there exists a surjective meromorphic map $\varphi: Y \rightarrow Z$ with the following commutative diagram.

(*)

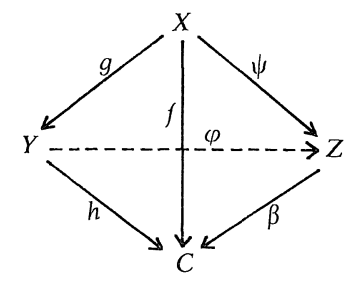

Proof. There is a commutative diagram.

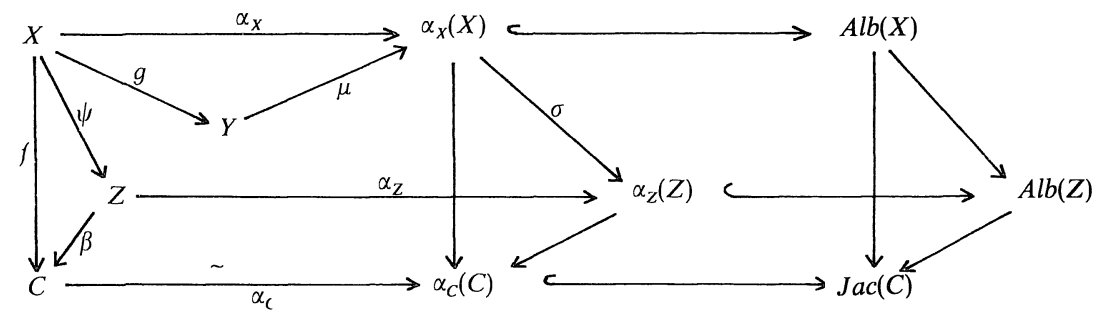

For a general fiber $X_{p}:=g^{-1}(p)(p \in Y)$ of $g$, we have

$$
\alpha_{Z^{\circ}} \psi\left(X_{p}\right)=\sigma \circ \alpha_{X}\left(X_{p}\right)=\sigma \circ \mu(p)=\{\text { one point }\} .
$$

Since $\alpha_{Z}$ is generically finite and $X_{p}$ is connected, $\psi\left(X_{p}\right)$ is a point for general $p \in Y$. Hence there exists a meromorphic map $\varphi: Y \cdots Z$ such that $\varphi \circ g$ is bimeromorphically equivalent to $\psi$ and $(*)$ holds.

q.e.d.

\section{§2. Proof of Theorem A}

Now we shall prove Theorem A.

Construction. There exists a finite Galois covering $\pi: \widetilde{C} \rightarrow C$ and an Abelian fiber space $\tilde{f}: \tilde{X} \rightarrow \widetilde{C}$ over $\tilde{C}$ which satisfies the following conditions.

1) The fiber space $\tilde{f}: \tilde{X} \rightarrow \widetilde{C}$ is bimeromorphically equivalent to $X \underset{C}{\times} \widetilde{C} \rightarrow \widetilde{C}$.

2) $\tilde{X}$ is a smooth projective manifold and $\tilde{f}$ is projective.

3) $\tilde{f}: \tilde{X} \rightarrow \widetilde{C}$ has a section. (So it is free from multile fibers.) 
4) $q(\tilde{f})$ is maximal among all finite Galois coverings $\widetilde{C} \rightarrow C$ of $C$. (In general we have $q(\tilde{f}) \leq \operatorname{dim}(X)$.)

Let

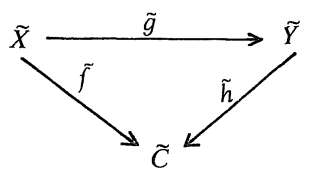

be the decomposition of Abelian fiber spaces as in Proposition 1.2. From the construction of $\tilde{Y}, \operatorname{Aut}(\tilde{X})$ induces $\operatorname{Aut}(\tilde{Y})$. Then if we put $G=\operatorname{Gal}(\tilde{X} / X)$, we have the following commutative diagram:

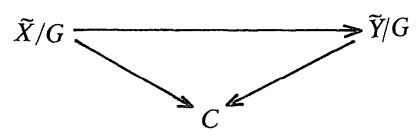

Taking a non-singular model of them, we obtain the commutative diagram

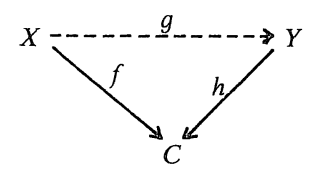

such that $g: X \ldots Y$ and $h: Y \rightarrow C$ are Abelian fiber spaces and the period map associated to $h$ is constant.

Universality. Suppose that there exists a commutative diagram such that the period map associated to $\beta$ is constant.

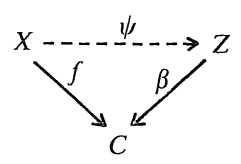

Then by Fujiki [1], Proposition 13.3, there exists a finite Galois covering $\widetilde{C} \rightarrow \widetilde{C}$ of $\widetilde{C}$ and by pulling-back $X \rightarrow Z \rightarrow C$ and taking a suitable bimeromorphic model, we obtain
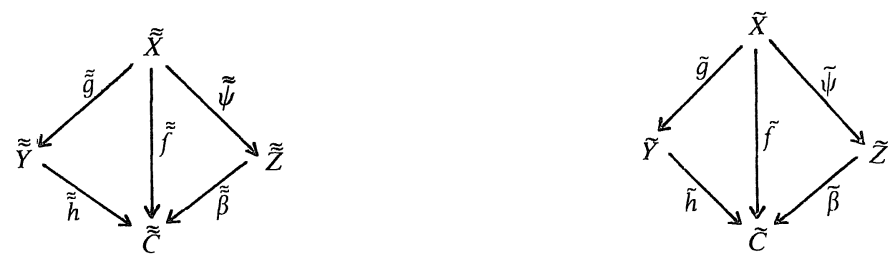
where $\tilde{Z} \rightarrow \tilde{\widetilde{C}}$ is bimeromorphic to $\tilde{C} \times B(B$ is an Abelian variety) and $\tilde{\psi}, \tilde{\psi}$ are morphisms. Then from the maximality of $q(\tilde{f})$, we have $q(\tilde{f})=q(\tilde{f})$. And clearly we have $q(\tilde{\tilde{f}}) \geq q(\tilde{\tilde{h}}) \geq q(\tilde{h})=q(\tilde{f})$, where the last equality holds from Proposition 1.2. Therefore we have $q(\tilde{\tilde{f}})=q(\tilde{h})$ and $q(\tilde{\widetilde{X}})=q(\tilde{\tilde{Y}})$.

Next we show that

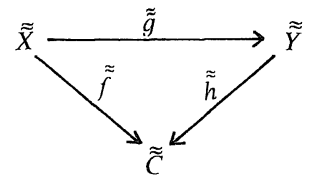

is obtained from the Stein factorization of the Albanese map of $\tilde{X}$. Let

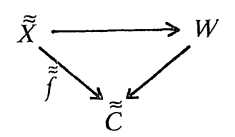

be the factorization as in Proposition 1.2. Then from the universality of $W$, there exists a unique morphism $\tilde{Y} \rightarrow W$ such that the following diagram is commutative.

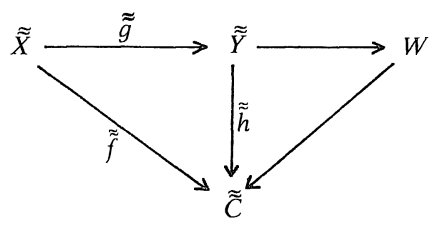

We have $\operatorname{dim}(\tilde{\tilde{Y}})=\operatorname{dim}(\tilde{Y})=1+q(\tilde{f})=1+q(\tilde{\tilde{f}})=\operatorname{dim}(W)$ from Proposition 1.2. Hence $\tilde{Y} \rightarrow W$ is a generically finite morphism. Since both $\tilde{\tilde{X}} \rightarrow \tilde{\widetilde{Y}}$ and $\tilde{\widetilde{X}}$ $\rightarrow W$ have connected fibers, $\tilde{Y}$ is bimeromorphic to $W$ and the claim has been proved.

By our construction, the Albanese map of $\tilde{\tilde{Z}}$. is birational to its image. So it follows from Lemma 1.3 that there exists a meromorphic map $\tilde{\bar{\varphi}}: \widetilde{\widetilde{Y}} \ldots, \widetilde{Z}$ with the following commutative diagram.

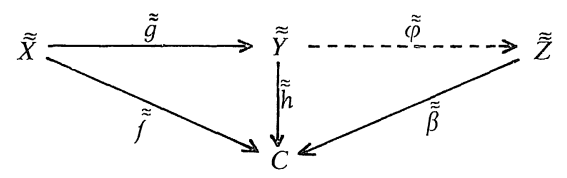

By the universality, the meromorphic map is compatible with the action of $G$ : = $\operatorname{Gal}(\tilde{X} / X)$. Hence $\tilde{\tilde{\varphi}}$ descends to a meromorphic map $\tilde{\varphi}: Y \cdots Z$ with the following commutative diagram. 


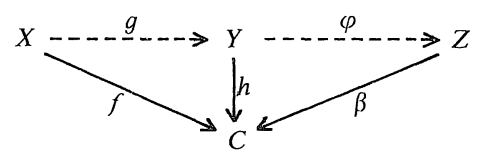

and the universality has been proved. Let

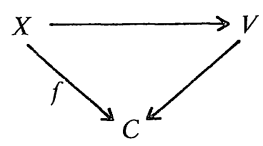

be a commutative diagam of Abelian fiber spaces as in Proposition 1.2. Then we have $q(V)=q(X)$ and $V \rightarrow C$ is a Seifert fiber space. Hence from the universality of $Y$, there exists a meromorphic map $Y \cdots \rightarrow$ with the commutative diagram.

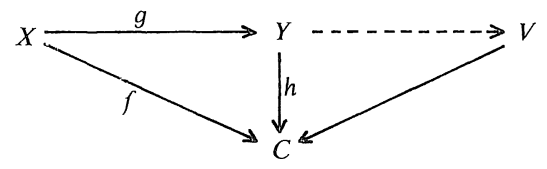

Clearly we have $q(X) \geq q(Y) \geq q(V)$. Since $q(X)=q(V)$, we have $q(X)=q(Y)$.

\section{References.}

[1] Fujiki, A., On the structure of compact complex manifolds in $\mathscr{C}$. Advanced studies in Pure Math. 1, Algebraic varieties and analytic varieties, 231-302, Kinokuniya, Tokyo (1983).

[2] Ueno, K., On algebraic fiber spaces of Abelian varieties. Math. Ann. 237 (1978), 1-22

[3] Zucker, S., Hodge theory with degenerating coefficients: $L^{2}$-cohomology in the Poincare metric. Annals of Math. 109 (1979), 415-476. 